\title{
Hepatoprotective effects of erythropoietin on D-galactosamine/lipopolysaccharide-induced fulminant hepatic failure in mice
}

\author{
XUE-FEI YANG*, YI HE* , HAI-YUAN LI, XIN LIU, HUAN CHEN, JIAN-BANG LIU, \\ WEN-JUN JI, BING WANG and LI-NA CHEN
}

Department of Pharmacology, College of Medicine, Xi'an Jiaotong University, Xi'an, Shaanxi 710061, P.R. China

Received July 24, 2013; Accepted March 24, 2014

DOI: $10.3892 / \mathrm{mmr} .2014 .2164$

\begin{abstract}
Fulminant hepatic failure is a severe clinical syndrome associated with a high rate of patient mortality. Recent studies have shown that in addition to its hematopoietic effect, erythropoietin (EPO) has multiple protective effects and exhibits antiapoptotic, antioxidant and anti-inflammatory activities. The present study aimed to determine the hepatoprotective effect of EPO and to elucidate the underlying mechanisms using a D-galactosamine (D-GalN)/lipopolysaccharide (LPS)-induced model of acute liver injury. Experimental groups of mice were administered with various doses of EPO $(1,000,3,000$ or $10,000 \mathrm{U} / \mathrm{kg}$, intraperitoneal) once per day for 3 days, prior to injection with D-GalN (700 mg/kg)/LPS (10 $\mu \mathrm{g} / \mathrm{kg})$. Mice were sacrificed $8 \mathrm{~h}$ after treatment with D-GalN/LPS. Liver function and histopathology, malondialdehyde (MDA), superoxide dismutase (SOD) and glutathione peroxidase (GSH-Px) activities and EPO receptor (EPOR) and phosphatidylinositol 3-kinase (PI3K) mRNA expression were evaluated. D-GalN/LPS administration markedly induced liver injury, as evidenced by elevated levels of serum aminotransferases, as well as histopathological changes. Compared with the D-GalN/LPS group, pretreatment with EPO significantly decreased the levels of aspartate aminotransferase, alanine aminotransferase and MDA, and increased
\end{abstract}

Correspondence to: Dr Li-Na Chen, Department of Pharmacology, College of Medicine, Xi'an Jiaotong University, 76 Yanta West Road, Xi'an, Shaanxi 710061, P.R. China

E-mail: chenlin@mail.xjtu.edu.cn

*Contributed equally

Abbreviations: FHF, fulminant hepatic failure; EPO, erythropoietin; D-GalN, D-galactosamine; LPS, lipopolysaccharide; MDA, malondialdehyde; SOD, superoxide dismutase; GSH-Px, glutathione peroxidase; EPOR, erythropoietin receptor; PI3K, phosphatidylinositol 3-kinase; AST, aspartate aminotransferase; ALT, alanine aminotransferase

Key words: erythropoietin, D-galactosamine/lipopolysaccharide, fulminant hepatic failure, hepatoprotective effects the activities of SOD and GSH-Px. Furthermore, the protective effects of EPO were paralleled by an upregulation in the mRNA expression of EPOR and PI3K. These data suggest that EPO can ameliorate D-GalN/LPS-induced acute liver injury by reducing oxidative stress and upregulating the mRNA expression of EPOR and PI3K.

\section{Introduction}

Fulminant hepatic failure (FHF) is a severe clinical syndrome that is characteristic of hepatic cell injury resulting from a variety of hepatic disease processes. FHF leads to hepatic encephalopathy, severe coagulopathy, jaundice, hydroperitoneum and high rates of patient mortality. Several mechanisms are responsible for FHF, including viral infection, drug or toxin intake and metabolic disorders (1). However, the pathophysiology of FHF remains poorly understood and further investigations are required. No therapy is currently available except liver transplantation, which is limited due to a shortage of donors, the rapid progression of FHF and the expense of the surgical procedure (2). Effective prophylactic or therapeutic interventions are urgently required to improve the prognosis of patients with FHF.

D-galactosamine (D-GalN) and lipopolysaccharide (LPS)-induced hepatic failure in mice is a universally used animal model that resembles acute hepatic failure in the clinic (3). D-GalN has been found to markedly strengthen the susceptibility of mice to the lethal effects of LPS (4). Upon stimulation with LPS, liver macrophages secrete various proinflammatory cytokines, leading to hepatic necrosis, decreased levels of antioxidant enzymes and the scavenging of free radicals $(5,6)$. Therefore, decreasing the generation of proinflammatory mediators and oxidative substances may be an effective strategy for the prevention or treatment of FHF.

Produced by interstitial fibroblasts in the kidney and in the fetal liver, erythropoietin (EPO) is an endogenous hormone that acts as a potent stimulator of bone marrow activity and red blood cell production (7). Although EPO is primarily synthesized in the kidney, it has been recognized that EPO and EPO receptors (EPORs) are expressed by other tissues and organs, including the brain, heart, kidney and liver (8-10). Furthermore, studies have revealed that EPO exerts antiapoptotic $(11,12)$, 
antioxidative (13-15) and anti-inflammatory (16) effects. Activation of the EPOR activates various intracellular pathways, including the mitogen-activated protein kinase, c-Jun $\mathrm{N}$-terminal kinase, nuclear factor $\mathrm{k}$-light-chain-enhancer of activated B cell and phosphatidylinositol 3-kinase (PI3K)/Akt signaling cascades $(17,18)$. In the present study, it was hypothesized that EPO may protect liver tissues against D-GalN and LPS-induced liver failure in mice. This study aimed to investigate this hypothesis.

\section{Materials and methods}

Materials. D-GalN and LPS were purchased from Sigma-Aldrich (St. Louis, MO, USA). Detection kits for alanine aminotransferase (ALT), aspartate aminotransferase (AST), superoxide dismutase (SOD), malondialdehyde (MDA) and glutathione peroxidase (GSH-Px) were purchased from Nanjing Jiancheng Bioengineering Institute (Nanjing, China).

Experimental animals, groups and treatment. Six- to eight-week-old BALB/c mice weighing between 18 and $22 \mathrm{~g}$ were obtained from the Experimental Animal Center of Xi'an Jiaotong University College of Medicine (Xi'an, China). All animals were acclimated to the laboratory environment for one week prior to the conduction of experimental procedures. The mice were allowed free access to drinking water and food and were maintained at $22 \pm 2^{\circ} \mathrm{C}$ in an automatic 12 -h light/dark cycle with $50 \%$ humidity. The study was approved by the Ethics Committee of Xi'an Jiaotong University and performed in accordance with the Practice Guidelines for Laboratory Animals of China.

The mice were randomly divided into the following five groups (n=8/group): Normal, D-GalN/LPS, and 1,000, 3,000 and $10,000 \mathrm{U} / \mathrm{kg}$ EPO. The dosages were selected based on previous investigations $(9,17)$ and a preliminary study. The EPO groups were administered EPO intraperitoneally once per day for 3 days. One hour after the final administration, mice in the normal group were injected with the same volume of sterile saline. The mice in the other group were simultaneously intraperitoneally injected with $700 \mathrm{mg} / \mathrm{kg} \mathrm{D}$-GalN and $10 \mu \mathrm{g} / \mathrm{kg}$ LPS. The mice were fasted for one night $(12 \mathrm{~h})$ prior to D-GalN and LPS administration. Eight hours after D-GalN and LPS injection, all mice were sacrificed under deep ether anesthesia. Blood samples were taken from the retro-orbital plexus of the mice. Serum was separated using centrifugation at $1,368 \mathrm{xg}$ for $10 \mathrm{~min}$, then stored at $-80^{\circ} \mathrm{C}$ for analysis of the serum levels of ALT and AST. Liver tissue samples were divided into two sections. One was immersed in $10 \%$ phosphate-buffered formalin for morphological analysis and the other was stored at $-80^{\circ} \mathrm{C}$ for the analysis of biochemical indicators of the liver.

Hepatic function tests. Serum AST and ALT levels were determined to assess liver function using AST and ALT commercial kits (Nanjing Jiancheng Bioengineering Institute).

MDA, SOD and GSH-Px assays. Liver tissue was washed with cold physiological saline and the connective tissue was removed. The liver tissue was then dried using filter paper and weighed. Tissue homogenate was prepared using cold physiological saline at a ratio of $1 / 9(\mathrm{w} / \mathrm{v})$, followed by centrifugation at $1,596 \mathrm{x}$ g for $10 \mathrm{~min}$. The supernatant was then collected and the concentration of MDA, and the activities of SOD and GSH-Px were measured using the corresponding kits according to the manufacturer's instructions (Nanjing Jiancheng Bioengineering Institute).

Histopathological analysis. Liver tissue was fixed in $10 \%$ neutral-buffered formalin and embedded in paraffin. The samples were sliced into $5-\mu \mathrm{m}$ sections and the slides were stained with hematoxylin and eosin.

RNA isolation and quantitative polymerase chain reaction $(q P C R)$ analysis. Total RNA was isolated from mouse liver tissues using the TriPure RNA isolation reagent (Roche, Basel, Switzerland) and $2 \mu \mathrm{g}$ RNA was reverse transcribed using the PrimeScript ${ }^{\mathrm{TM}}$ RT Master Mix (Perfect Real Time) (Takara Bio, Inc., Tokyo, Japan). qPCR analysis was performed using SYBR $^{\circledR}$ Premix Ex Taq ${ }^{\mathrm{TM}}$ II (Perfect Real Time) (Takara Bio, Inc.). PCR reactions were performed in 96-well plates in an iQ5 Real-Time PCR Detection system (Bio-Rad Laboratories, Hercules, CA, USA).

All of the primers and probes for qPCR were obtained from Takara Bio, Inc. Murine EPOR was amplified using the primers EPOR-F (5'-GCT CCG GGA TGG ACT TCA ACT A-3') and EPOR-R (5'-CTG GTG CAG GCT ACA TGA CTT TC-3'); murine PI3K was amplified using the primers PIK3R1-F (5'-GCT CCT GGA AGC CAT TGA GAA-3') and PIK3R1-R (5'-CGT CGA TCA TCT CCA AGT CCA C-3'); and murine GAPDH was amplified using the primers GAPDH-F (5'-TGT GTC CGT CGT GGA TCT GA-3') and GAPDH-R (5'-TTG CTG TTG AAG TCG CAG GAG-3'). GAPDH served as an endogenous control and the results were normalized to GAPDH. The efficiency of qPCR for the target genes and the endogenous control was approximately equal. $-\Delta \mathrm{CT}$ expresses the difference between the number of cycles (CT) of the target genes and the endogenous control. Results are expressed as $2^{-\Delta \Delta C T}$ and show the fold increase in gene expression compared with the control group. Bio-Rad iQ5 software (Bio-Rad Laboratories) was used to perform the data analysis and generate the standard curve.

Statistical analysis. All data are presented as the mean \pm standard error of the mean. The results were evaluated using one-way analysis of variance and Tukey's multiple comparison tests. A value of $\mathrm{P}<0.05$ was considered to indicate a statistically significant difference.

\section{Results}

Effect of EPO on serum levels of ALT and AST in a $D$-GalN/LPS-induced model of FHF. ALT and AST are two important indicators of liver function. As shown in Fig. 1, ALT and AST levels were observed to be significantly elevated in the mice in the D-GalN/LPS group compared with those in the normal group $(\mathrm{P}<0.01)$, indicating severe liver injury in the D-GalN/LPS mice. Pretreatment with various doses of EPO was found to reduce the increases in ALT and AST by varying degrees. In the mice in the $10,000 \mathrm{U} / \mathrm{kg}$ EPO group, serum AST and ALT activities were significantly reduced compared with activities in the D-GalN/LPS group $(\mathrm{P}<0.01)$. 
A

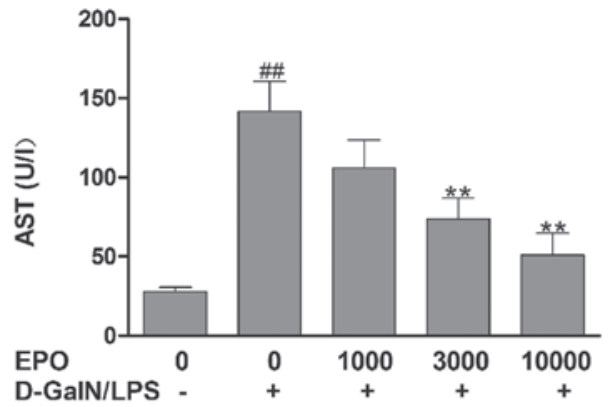

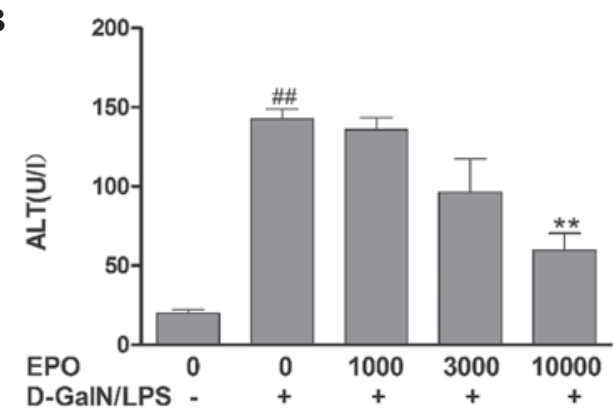

Figure 1. Effect of EPO on serum (A) AST and (B) ALT levels. Mice were pretreated with 1,000,3,000 and 10,000 U/kg EPO per day for 3 days and $1 \mathrm{~h}$ prior to D-GalN/LPS injecton. After $8 \mathrm{~h}$, blood samples were collected. Data are presented as the mean \pm standard error of the mean $(\mathrm{n}=8)$. ${ }^{\# \#} \mathrm{P}<0.01 \mathrm{vs}$. normal group; ${ }^{* *} \mathrm{P}<0.01$ vs. D-GalN/LPS group. EPO, erythropoietin; ALT, alanine aminotransferase; AST, aspartate aminotransferase; D-GalN, D-galactosamine; LPS, lipopolysaccharide.

A

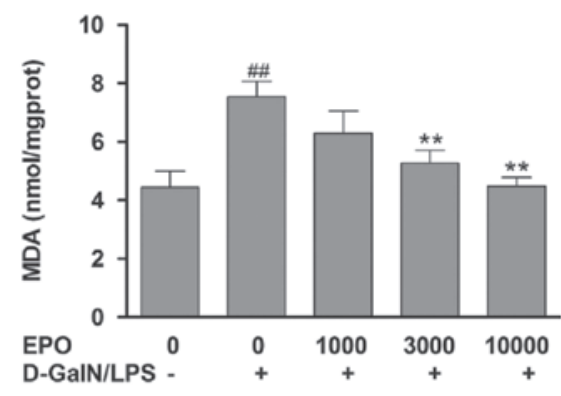

B

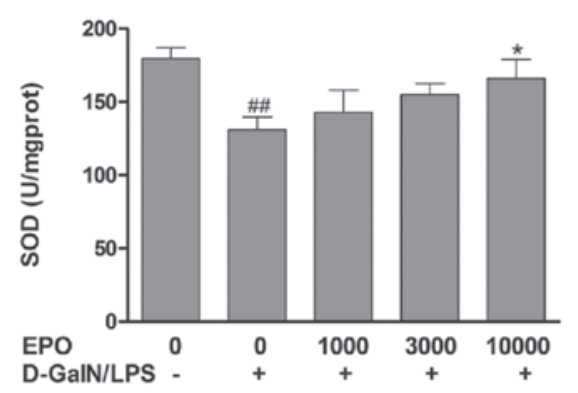

C

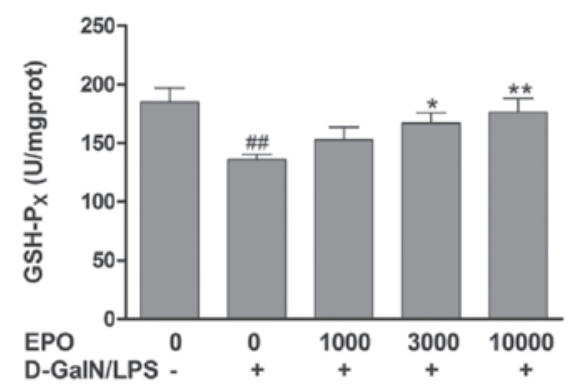

Figure 2. Effect of EPO on MDA, SOD and GSH-Px levels. Mice were pretreated with 1,000, 3,000 and 10,000 U/kg EPO per day for 3 days and $1 \mathrm{~h}$ prior to D-GalN/LPS injecton. After $8 \mathrm{~h}$, liver tissue was collected. (A-C) Effect of EPO on (A) MDA content; (B) SOD activity; (C) GSH-Px activity. Data are presented as the mean \pm standard error of the mean $(n=8)$. ${ }^{\# *} \mathrm{P}<0.01$ vs. normal group; ${ }^{*} \mathrm{P}<0.05$ and ${ }^{* *} \mathrm{P}<0.01$ vs. D-GalN/LPS group. EPO, erythropoietin; MDA, malondialdehyde; SOD, superoxide dismutase; GSH-Px, glutathione peroxidase; D-GalN, D-galactosamine; LPS, lipopolysaccharide.

Effect of EPO on MDA, SOD and GSH-Px levels. As indicated in Fig. 2, $8 \mathrm{~h}$ after D-GalN/LPS administration, the concentration of MDA, a marker of free radical-induced injury, was determined. The activities of the antioxidant enzymes SOD and GSH-Px were also analyzed. Liver tissue MDA levels were increased significantly in the D-GalN/LPS group compared with those in the normal group $(\mathrm{P}<0.01)$. However, the activities of SOD and GSH-Px were markedly reduced in the mice in the D-GalN/LPS group compared with those in the normal group $(\mathrm{P}<0.01)$. The elevation in MDA was significantly attenuated with 3,000 and 10,000 U/kg EPO compared with the D-GalN/LPS group $(\mathrm{P}<0.01)$. Furthermore, EPO pretreatment was found to enhance the activities of SOD and GSH-Px.

EPO alleviates liver injury in D-GalN/LPS-induced FHF mice. In the normal group, the hematoxylin and eosin-stained liver sections exhibited an integrated architecture of hepatic lobules and normal cell structure without necrosis (Fig. 3). However, injection with D-GalN/LPS was found to induce destruction of the liver structure, severe hepatocyte necrosis, hemorrhage and inflammatory cell infiltration. Liver tissue damage was ameliorated in the mice pretreated with EPO, compared with those in the D-GalN/LPS group.

Effects of EPO on the expression of EPOR and PI3K. In order to further investigate the mechanism underlying the effect of
EPO on FHF, the hepatic expression of EPOR and PI3K was assessed in the different groups. The expression of EPOR and PI3K was calculated relative to that of the endogenous control, GAPDH. As shown in Fig. 4, the expression of EPOR and PI3K in the mice in the D-GalN/LPS group was significantly decreased compared with that in the normal group $(\mathrm{P}<0.05)$. However, pretreatment with EPO was found to significantly increase the mRNA expression of EPOR and PI3K mRNA in comparison with the D-GalN/LPS group $(\mathrm{P}<0.05)$.

\section{Discussion}

D-GalN/LPS-treated mouse models are frequently used to study the pathogenesis of liver injury (19-21). D-GalN is a specific hepatotoxin that selectively depletes uridine nucleotides, ultimately inhibiting macromolecule synthesis in hepatocytes, which results in abnormalities in the structure and function of hepatic cells. In addition, D-GalN may act synergistically with LPS. Activated neutrophils, which accumulate around damaged liver cells, undergo bursts of respiration and degranulation, releasing oxygen free radicals and leading to lipid peroxidation. Oxygen free radicals and lipid peroxidation target liver parenchyma and vascular endothelial cells, resulting in cell damage or death $(22,23)$. In the present study, intraperitoneal injection of D-GalN/LPS in mice resulted in severe hepatic injury, which was associated with elevated serum activity of AST and ALT. 

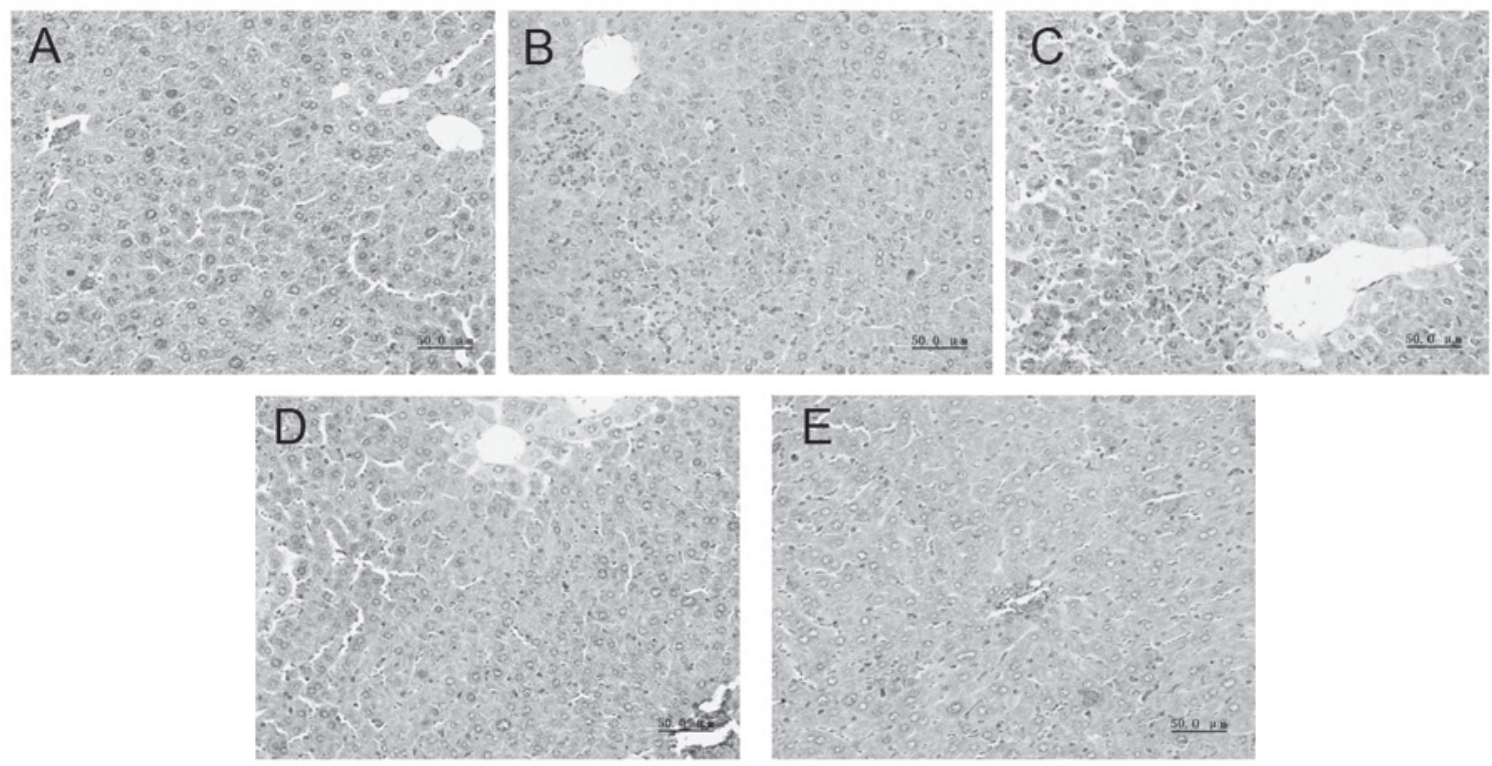

Figure 3. Morphological analysis of the liver tissue using hematoxylin and eosin staining. Mice were pretreated with 1,000, 3,000 and 10,000 U/kg EPO per day for 3 days and $1 \mathrm{~h}$ prior to D-GalN/LPS injecton. After $8 \mathrm{~h}$, liver tissue was collected and subjected to hematoxylin and eosin staining. (A) Normal group, (B) D-GalN/LPS group, (C) EPO (1,000 U/kg)+D-GalN/LPS group, (D) EPO (3,000 U/kg)+D-GalN/LPS group, (E) EPO (10,000 U/kg)+D-GalN/LPS group (magnification, x400). EPO, erythropoietin; D-GalN, D-galactosamine; LPS, lipopolysaccharide.

A

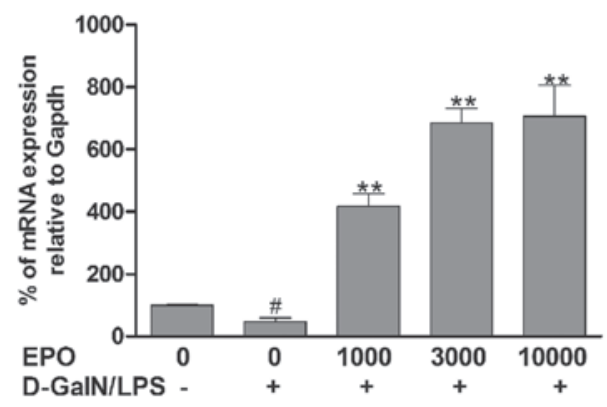

B

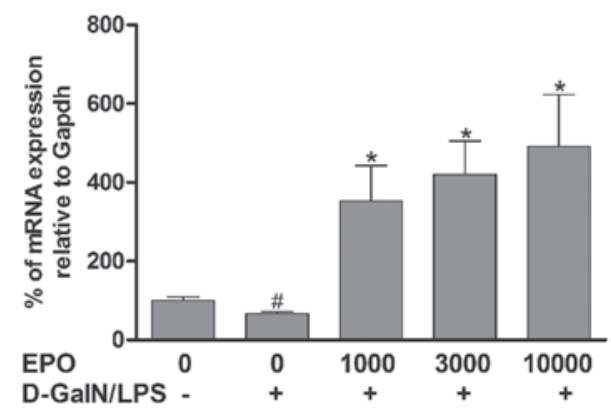

Figure 4. Effect of EPO on EPOR and PI3K gene expression in mice with D-GalN/LPS-induced liver injury. (A and B) Effect of EPO on (A) EPOR and (B) PI3K gene expression. Data are presented as the mean \pm standard error of the mean $(\mathrm{n}=8) .{ }^{*} \mathrm{P}<0.05$ vs. normal group; ${ }^{*} \mathrm{P}<0.05$ vs. $\mathrm{D}$ GalN/LPS group, ${ }^{* *} \mathrm{P}<0.01$ vs. D-GalN/LPS group. EPO, erythropoietin; EPOR, EPO receptor; PI3K, phosphatidylinositol 3-kinase; D-GalN, D-galactosamine; LPS, lipopolysaccharide.

Histopathological analysis also showed that D-GalN/LPS induced severe necrosis in hepatocytes, hemorrhage and inflammatory cell infiltration. Reactive oxygen species have a major role in the initiation of D-GalN/LPS-induced liver injury $(24,25)$, which is consistent with the findings of the present study. MDA, a marker of lipid peroxidation, was observed to be present in significantly higher levels in the liver tissue of mice in the D-GalN/LPS group compared with mice in the normal group, and the activity of SOD and GSH-Px, antioxidant markers, was significantly lower.

The present study showed that, in the mice pretreated with EPO, the serum activity of AST and ALT was decreased and the degree of liver tissue damage was ameliorated, compared with mice in the D-GalN/LPS group. This suggests that EPO has a strong hepatoprotective effect on D-GalN/LPS-induced liver injury. EPO has been shown to exert antioxidative effects against hypoxic-ischemic brain injury (26) and chemical toxins (27). In the present study, intraperitoneal administration of EPO decreased the level of MDA and increased the activity of antioxidant enzymes, including SOD and GSH-Px, in the liver tissue compared with the D-GalN/LPS group. These findings are in accordance with those of other in vivo studies (28-30) and the histopathological observations.

EPO has been proposed to be a tissue-protective hormone with pleiotropic potential. EPO exerts protective effects against tissue destruction surrounding sites of injury by signaling through a nonhemopoietic receptor (31). In addition, when exerting its extra-hematopoietic effects, EPO has been shown to bind to heterodimeric EPOR, which consists of EPOR and the $\beta$ common receptor (32). One of the most important signaling pathways is the PI3K/Akt pathway. The PI3K/Akt signaling pathway is an important pathway for survival, and protects the body against various stresses. The activation of the PI3K/Akt pathway regulates cell apoptosis, survival and proliferation through the regulation of its downstream factors. Studies have shown that this pathway has a positive protective effect in liver ischemia-reperfusion injury (33-35). Furthermore, another study found that EPO has a protective effect against injury in 
proximal tubule cells and cardiac myocytes by activating EPOR, which leads to activation of PI3K and ultimately Akt (36). Thus, it was of interest to examine the effect of EPO pretreatment on the PI3K/Akt pathway in D-GalN/LPS-induced acute liver failure in a murine model. The present study showed that EPOR and PI3K gene expression was significantly decreased in D-GalN/LPS mice, while pretreatment with EPO significantly increased EPOR and PI3K mRNA expression in a dose-dependent manner. Therefore, EPO may exert its hepatoprotective effects through upregulation of EPOR and PI3K.

In conclusion, to the best of our knowledge, the results of the present study are the first to demonstrate a protective effect of exogenous EPO against D-GalN/LPS-induced hepatic injury. In addition, the mechanism underlying the protective effect of EPO has been shown to be associated with antioxidant properties and the upregulation of EPOR and PI3K expression. These findings may contribute to the development of novel agents for the treatment of FHF. However, further investigations are required to determine the precise protective mechanism.

\section{Acknowledgements}

This study was supported by grants from the National Natural Science Youth Foundation of China (no. 30901808), the Technology and Innovation Project of Shaanxi Province (no. 2011KTCL03-20) and the National College Students Innovation Training Project (no. 201210698130).

\section{References}

1. Schiødt FV and Lee WM: Fulminant liver disease. Clin Liver Dis 7: 331-349, 2003.

2. Mukherjee S, Mahmoudi TM and Mukherjee U: Liver transplant for viral hepatitis and fulminant hepatic failure. Minerva Gastroenterol Dietol 55: 83-100, 2009.

3. Xing WW, Zou MJ, Liu S, Xu T, Gao J, Wang JX and Xu DG: Hepatoprotective effects of IL-22 on fulminant hepatic failure induced by d-galactosamine and lipopolysaccharide in mice. Cytokine 56: 174-179, 2011

4. Shin JW, Wang JH, Park HJ, Choi MK, Kim HG and Son CG: Herbal formula CGX ameliorates LPS/D-galactosamine-induced hepatitis. Food Chem Toxicol 49: 1329-1334, 2011.

5. Jeong YI, Jung ID, Lee CM, et al: The novel role of platelet-activating factor in protecting mice against lipopolysaccharide-induced endotoxic shock. PLoS One 4: e6503, 2009.

6. Thirunavukkarasu C, Uemura T, Wang LF, Watkins SC and Gandhi CR: Normal rat hepatic stellate cells respond to endotoxin in LBP-independent manner to produce inhibitor(s) of DNA synthesis in hepatocytes. J Cell Physiol 204: 654-665, 2005.

7. Fisher JW: Erythropoietin: physiology and pharmacology update. Exp Biol Med (Maywood) 228: 1-14, 2003.

8. Coleman T and Brines M: Science review: recombinant human erythropoietin in critical illness: a role beyond anemia? Crit Care 8: 337-341, 2004.

9. Sepodes B, Maio R, Pinto R, et al: Recombinant human erythropoietin protects the liver from hepatic ischemia-reperfusion injury in the rat. Transpl Int 19: 919-926, 2006.

10. Lewis LD: Preclinical and clinical studies: a preview of potential future applications of erythropoietic agents. Semin Hematol 41 (4 Suppl 7): 17-25, 2004.

11. Kadota T, Shingo T, Yasuhara T, et al: Continuous intraventricular infusion of erythropoietin exerts neuroprotective/rescue effects upon Parkinson's disease model of rats with enhanced neurogenesis. Brain Res 1254: 120-127, 2009.

12. Mammis A, McIntosh TK and Maniker AH: Erythropoietin as a neuroprotective agent in traumatic brain injury Review. Surg Neurol 71: 527-531, 2009.

13. Lippi G, Franchini M and Banfi G: Biochemistry and physiology of anabolic androgenic steroids doping. Mini Rev Med Chem 11: 362-373, 2011.
14. Dimitrijevic ZM, Cvetkovic TP, Djordjevic VM, et al: How the duration period of erythropoietin treatment influences the oxidative status of hemodialysis patients. Int J Med Sci 9: 808-815, 2012.

15. Akisu M, Küllahçioğlu Girgin F, Baka M, Hüsseyinov A and Kültürsay N: The role of recombinant human erythropoietin in lipid peroxidation and platelet-activating factor generation in a rat model of necrotizing enterocolitis. Eur J Pediatr Surg 11: 167-172, 2001.

16. Nairz M, Sonnweber T, Schroll A, Theurl I and Weiss G: The pleiotropic effects of erythropoietin in infection and inflammation. Microbes Infect 14: 238-246, 2012.

17. Yang CW, Li C, Jung JY, et al: Preconditioning with erythropoietin protects against subsequent ischemia-reperfusion injury in rat kidney. FASEB J 17: 1754-1755, 2003.

18. Chong ZZ, Kang JQ and Maiese K: Hematopoietic factor erythropoietin fosters neuroprotection through novel signal transduction cascades. J Cereb Blood Flow Metab 22: 503-514, 2002.

19. Kudo H, Takahara T, Yata Y, Kawai K, Zhang W and Sugiyama T: Lipopolysaccharide triggered TNF-alpha-induced hepatocyte apoptosis in a murine non-alcoholic steatohepatitis model. J Hepatol 51: 168-175, 2009.

20. Kuhla A, Eipel C, Abshagen K, Siebert N, Menger MD and Vollmar B: Role of the perforin/granzyme cell death pathway in D-Gal/LPS-induced inflammatory liver injury. Am J Physiol Gastrointest Liver Physiol 296: G1069-G1076, 2009.

21. Ikeda T, Abe K, Kuroda N, et al: The inhibition of apoptosis by glycyrrhizin in hepatic injury induced by injection of lipopolysaccharide/D-galactosamine in mice. Arch Histol Cytol 71: 163-178, 2008.

22. Chen JC, Ng CJ, Chiu TF and Chen HM: Altered neutrophil apoptosis activity is reversed by melatonin in liver ischemia-reperfusion. J Pineal Res 34: 260-264, 2003.

23. Jaeschke H: Reactive oxygen and mechanisms of inflammatory liver injury: Present concepts. J Gastroenterol Hepatol 26 (Suppl 1): 173-179, 2011.

24. Han D, Hanawa N, Saberi B and Kaplowitz N: Hydrogen peroxide and redox modulation sensitize primary mouse hepatocytes to TNF-induced apoptosis. Free Radic Biol Med 41: 627-639, 2006

25. Zou W, Roth RA, Younis HS, Burgoon LD and Ganey PE: Oxidative stress is important in the pathogenesis of liver injury induced by sulindac and lipopolysaccharide cotreatment. Toxicology 272: 32-38, 2010.

26. Fan X and van Bel F: Pharmacological neuroprotection after perinatal asphyxia. J Matern Fetal Neonatal Med 23 (Suppl 3): 17-19, 2010.

27. Shang Y, Li X, Prasad PV, et al: Erythropoietin attenuates lung injury in lipopolysaccharide treated rats. J Surg Res 155: 104-110, 2009.

28. Cetin H, Olgar S, Oktem F, Ciris M, Uz E, Aslan C and Ozguner F: Novel evidence suggesting an anti-oxidant property for erythropoietin on vancomycin-induced nephrotoxicity in a rat model. Clin Exp Pharmacol Physiol 34: 1181-1185, 2007.

29. Guneli E, Cavdar Z, Islekel H, et al: Erythropoietin protects the intestine against ischemia/reperfusion injury in rats. Mol Med 13: 509-517, 2007.

30. Oztürk E, Demirbilek S, Köroğlu A, et al: Propofol and erythropoietin antioxidant properties in rat brain injured tissue. Prog in Neuropsychopharmacol Biol Psychiatry 32: 81-86, 2008.

31. Brines $M$ and Cerami A: Emerging biological roles for erythropoietin in the nervous system. Nat Rev Neurosci 6: 484-494, 2005.

32. Brines M, Grasso G, Fiordaliso F, et al: Erythropoietin mediates tissue protection through an erythropoietin and common beta-subunit heteroreceptor. Proc Natl Acad Sci USA 101: 14907-14912, 2004.

33. Datta SR, Brunet A and Greenberg ME: Cellular survival: a play in three Akts. Genes Dev 13: 2905-2927, 1999.

34. Meier R, Alessi DR, Cron P, Andjelković M and Hemmings BA: Mitogenic activation, phosphorylation, and nuclear translocation of protein kinase Bbeta. J Biol Chem 272: 30491-30497, 1997.

35. Kim AH, Khursigara G, Sun X, Franke TF and Chao MV: Akt phosphorylates and negatively regulates apoptosis signal-regulating kinase 1. Mol Cell Biol 21: 893-901, 2001.

36. Abdelrahman M, Sharples EJ, McDonald MC, Collin M, Patel NS, Yaqoob MM and Thiemermann C: Erythropoietin attenuates the tissue injury associated with hemorrhagic shock and myocardial ischemia. Shock 22: 63-69, 2004. 